\title{
A Comprehensive Study on Next-Generation Optical Grooming Switches
}

\author{
Keyao Zhu, Student Member, IEEE, Hui Zang, Member, IEEE, and Biswanath Mukherjee, Member, IEEE
}

\begin{abstract}
This paper investigates the characteristics and performance of different optical grooming switches, i.e., optical cross-connects (OXCs) capable of traffic grooming, under a dynamic traffic environment. We present four optical grooming-OXC architectures, namely, single-hop grooming $\mathrm{OXC}$, multihop partial-grooming $\mathrm{OXC}$, multihop full-grooming $\mathrm{OXC}$, and light-tree-based source-node grooming OXC. After exploring their grooming capabilities, we propose three grooming schemes and two corresponding algorithms, grooming using auxiliary graph and grooming using light-tree. Through the algorithms, we evaluate the performance of different optical grooming OXCs in a dynamic traffic environment under different connection bandwidth-granularity distributions. Our investigation uncovers the following results: 1) the multihop full-grooming OXC can achieve the best network performance, but it may encounter cost and scalability constraints; 2) by using significantly less low-granularity electronic processing and intelligent traffic-grooming algorithms, the multihop partial-grooming OXC shows reasonable network performance and, hence, can be viewed as a cost-effective alternative when a network node does not require full-grooming capability; 3) the single-hop grooming OXC may cause a large amount of capacity waste and lead to poor network performance; and 4) through its multicast capability, a light-tree-based source-node grooming OXC can significantly out-perform the performance of a single-hop grooming $\mathrm{OXC}$ in terms of network throughput and network resource efficiency. From our results, we also observe that the connection bandwidth-granularity distribution has a large impact on network throughput and network resource efficiency and, therefore, should be carefully considered for network design and traffic provisioning.
\end{abstract}

Index Terms-Grooming switch, lightpath, light tree, multigranularity, optical, optical cross-connects (OXCs), traffic grooming, wavelength-division multiplexing (WDM).

\section{INTRODUCTION}

\section{A. Next-Generation Optical WDM Networks}

$\mathbf{F}$ IBER optics and wavelength-division multiplexing (WDM) technology have significantly increased the transmission capacity of today's transport networks, and they are playing important roles in supporting the rapidly increasing data traffic. Using WDM, the bandwidth of a fiber link can be divided into tens (or hundreds) of nonoverlaping wavelength

Manuscript received August 1, 2002; revised March 2003. This work was supported in part by the National Science Foundation (NSF) under Grant NCR9508239 and Grant ANI-9805285, and in part by Sprint Advanced Technology Laboratories (ATL). This paper was presented in part at the IEEE/OSA Optical Fiber Communication Conference (OFC), Anaheim, CA, March 2002.

K. Zhu and B. Mukherjee are with the Department of Computer Science, University of California, Davis, CA 95616 USA (e-mail: zhuk@ cs.ucdavis.edu; mukherje@cs.ucdavis.edu).

H. Zang is with Sprint Advanced Technology Laboratories, Burlingame, CA 94010 USA (e-mail: hzang@ sprintlabs.com).

Digital Object Identifier 10.1109/JSAC.2003.815683 channels (i.e., frequency channels), each of which can operate at peak electronic processing rate, i.e., over a few gigabits per second $(\mathrm{Gb} / \mathrm{s})$. The bandwidth of a wavelength channel can be further divided into finer bandwidth-granularity trunks using the time-division multiplexing (TDM) technique, thus, a wavelength channel can be shared by different end users. An end user can be any type of client network equipment such as IP, ATM or frame relay network equipment. Hence, an optical WDM network serves as an important platform (namely, a circuit core) to provide network connectivity as well as transmission capacity to today's Internet infrastructure and application services [1], [2].

As WDM switching technology keeps maturing, optical WDM networks are expected to evolve from interconnected synchronous optical network (SONET)/WDM ring topologies to arbitrary-mesh topologies, and network provisioning will migrate from an on-site manual interconnection process to a point-and-click or on-demand automatic switching and connection process. Such an intelligent optical WDM network is emerging under the joint efforts of optical switch [or optical cross-connects (OXCs)] development, optical network control plane standardization, and extensive optical WDM network research and experiment activities in industry and academe. Among different optical WDM switching technologies (i.e., circuit switching, burst switching, and packet switching), WDM circuit switching is believed to be the most practical approach to enable the next-generation optical network. Consequently, this study concentrates on an intelligent optical circuit-switched WDM transport network.

\section{B. Traffic Grooming in Next-Generation Optical WDM Networks}

Traffic grooming is a procedure of efficiently multiplexing/ demultiplexing and switching low-speed traffic streams onto/ from high-capacity bandwidth trunks in order to improve bandwidth utilization, optimize network throughput, and minimize network cost. Although the grooming concept has been existing in telecommunication industry for years (e.g., DS0 to T1, T1 to SONET STS-1, etc.), because of the lack of intelligent network control and automatic provisioning functionality, traditional traffic grooming is more like a multiplexing/demultiplexing concept rather than an efficient, hierarchical, multigranularity switching and end-to-end provisioning concept. Traffic grooming is an extremely important issue for next-generation optical WDM networks to cost-effectively perform end-to-end automatic provisioning. In different optical WDM network domains, different multiplexing technologies may be applied for traffic grooming. For example, the TDM scheme can be 
used to perform time-slot to wavelength channel grooming in a SONET/SDH-over-WDM network environment; WDM scheme can be used to perform wavelength channel to waveband grooming or wavelength/waveband to fiber grooming in an all-optical WDM network environment ${ }^{1}$; and packet-based statistical multiplexing scheme can be used to perform packet flow or virtual circuit (VC) to wavelength channel grooming in an IP-over-WDM network environment, etc. Different multiplexing techniques may impose different grooming constraints. In this paper, we consider a hybrid TDM-over-WDM (SONET/WDM) based optical network environment, in which $\mathrm{OXCs}^{2}$ with different architectures are used for constructing an intelligent next-generation optical core network.

\section{Related Studies and Our Contribution}

Most of the earlier traffic-grooming research focused on network design and optimization for SONET/WDM ring networks [3]-[9]. By employing wavelength add-drop multiplexers (W-ADMs), efficient wavelength assignment and time-slot assignment algorithms have been investigated to design a SONET/WDM ring network such that all traffic requests can be accommodated and, simultaneously, the network cost [which is dominated by electrical SONET add-drop multiplexers (SONET-ADMs)] can be minimized.

In recent years, as optical transport networks keep evolving from interconnected SONET/WDM ring networks to arbitrary-mesh-based optical WDM networks, increasing amount of research efforts have been conducted on the traffic-grooming problem in optical WDM mesh networks. The authors in [10] studied the network design and traffic optimization problem with grooming consideration. Two grooming-node architectures in WDM mesh networks were presented. The problem was formulated as an integer linear program (ILP), and two heuristic algorithms were proposed as well. The authors in [11] quantitively showed the network-cost gain by employing grooming capability in optical core networks for static traffic demands. A generic graph model was presented for provisioning multigranularity connections in multiwavelength optical WDM networks in [12]. The authors showed that different network optimization objectives and corresponding route-selection schemes can be easily accommodated by this graph model. Based on this model, the authors proposed several grooming heuristics for static traffic demands and showed that these heuristics can achieve near-optimal solutions. The work in [13]-[17] considered traffic-grooming issues in dynamic traffic environment. The authors in [13] used the layered-graph model proposed in [12] to show how to efficiently low-speed connections according to different traffic-engineering objectives. The authors in [14] observed that, in a multigranularity WDM network, it is more possible to block connections with high-bandwidth requirement than to block those with low-bandwidth requirement, which results in unfairness between connections of different bandwidth-granularity classes. Hence, they proposed a call admission control (CRC) algorithm

\footnotetext{
${ }^{1}$ An all-optical WDM network can switch traffic in the optical domain without converting optical signals to electronic signals.

${ }^{2}$ Note that an OXC is known as an intelligent optical switch. We use these two terminologies interchangeably in this paper.
}

to achieve the fairness. The authors in [15] and [16] studied on-line provisioning mechanisms for connections of different bandwidth granularities in a traffic-groomable WDM network. Several on-line algorithms were proposed to optimize the overall network performance. The authors in [17] proposed a generalized network model, namely, trunk switched network (TSN), to facilitate the modeling and analysis of a multiwavelength TDM-switched network. They analyzed the blocking performance of TSN and extended their model to analyze the blocking performance of multicast-tree establishment in optical WDM networks.

In this paper, we systematically investigate and evaluate the characteristics of different optical grooming switches, i.e., the OXCs with traffic-grooming capability. According to various OXC architectures, we explore and propose different traffic-grooming schemes, and compare the performance of the grooming OXCs and grooming schemes under a dynamic traffic environment. To the best of our knowledge, this is one of the first studies which comprehensively examines the characteristics and performance of different types of optical grooming OXCs for dynamic traffic under different connection bandwidth-granularity distributions. Our investigation should assist network operators to cost-effectively design and operate an optical groomable WDM backbone network and it will be also helpful for equipment vendors to develop high-performance grooming OXCs.

\section{Organization}

The rest of the paper is organized as follows. In Section II, we introduce the architectures of different grooming OXCs and explore their corresponding grooming schemes. Section III proposes the detailed approaches and algorithms for the different grooming schemes using different grooming OXCs. Illustrative numerical results are presented and analyzed in Section IV. Section V concludes the paper.

\section{DifFERENT GROOMING Switch ARChiteCtURES AND CORRESPONDING GROOMING SCHEMES}

In an optical WDM network, a lightpath [1], [2] provides a basic communication mechanism between two network nodes. From traffic-grooming perspective, a lightpath is a circuit with full wavelength capacity. It may span one or multiple fiber links and be switched by intermediate nodes. Low-speed traffic streams can be packed onto a lightpath at its end nodes by grooming OXCs. There are transparent (all-optical) or opaque switching technologies to implement those OXCs. Transparent (all-optical) technology refers to the switching without optical-to-electronic (OE) conversion. Opaque technology refers to the switching with OE conversion. Different technologies and architectures may lead to different grooming OXCs, which may be capable of different grooming schemes. Specifically, there are three grooming schemes, namely, single-hop grooming, multihop grooming, and light-tree-based source-node grooming. Each grooming OXC may support one or more grooming schemes. Different types of grooming OXCs with their corresponding grooming schemes are categorized in the following sections. 


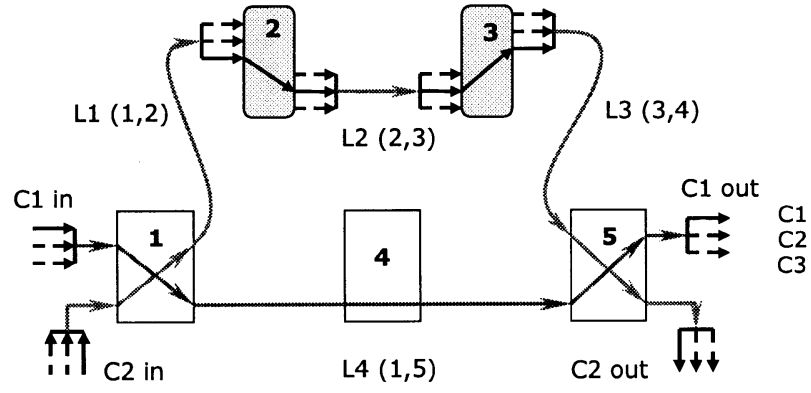

(a)

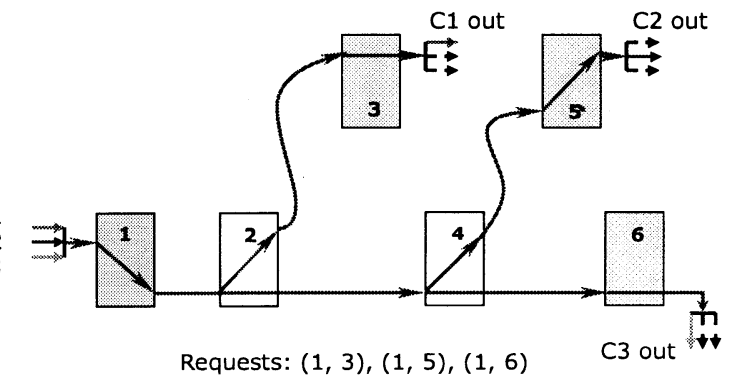

(b)

Fig. 1. Examples of single-hop, multihop, and source-node grooming schemes.

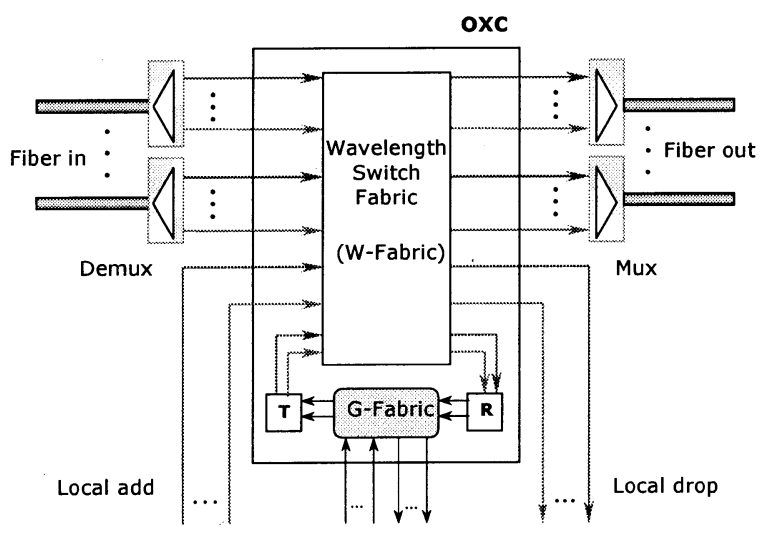

(a)

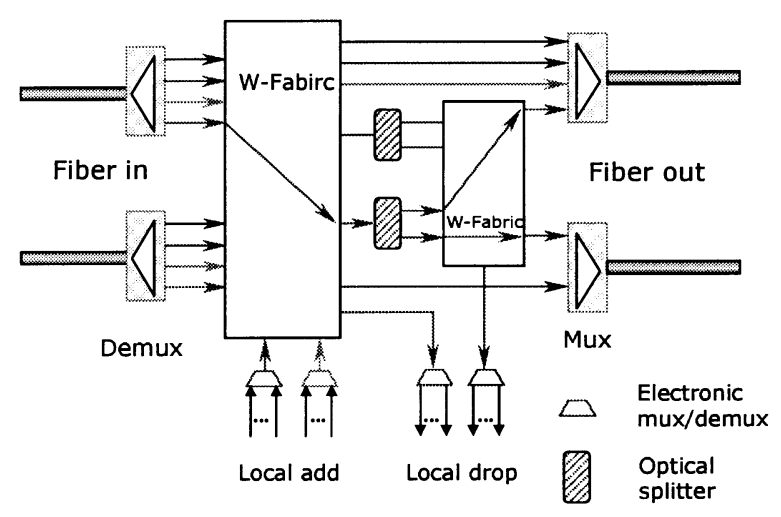

(b)

Fig. 2. Sample grooming OXC architectures: a multihop partial-grooming OXC and a source-node grooming OXC.

\section{A. Single-Hop Grooming OXC}

An OXC is called a single-hop grooming OXC if 1) it can only switch at wavelength granularity and 2) it has low-data-rate interfaces (ports) which can directly support low-speed traffic streams from client network elements. Note that cooperating with a separated network aggregation equipment (e.g., an electrical multiplexer), an OXC with only wavelength-granularity input/output ports and only switching at wavelength granularity is equivalent to and can be viewed as a single-hop grooming OXC. Using this type of OXCs, low-speed traffic from client equipment can be multiplexed onto a wavelength channel using a TDM scheme. Since this type of OXCs does not have the capability of switching low-granularity traffic at intermediate nodes, all of the low-speed streams on one wavelength channel at the source node will be switched to the same destination node, i.e., a low-speed connection can only traverse a single-lightpath hop. Therefore, this end-to-end grooming scheme is called single-hop grooming scheme.

Fig. 1(a) shows how a low-speed connection $(C 1)$ is carried by a lightpath $(L 4)$ from node 1 to node 5 using the single-hop grooming scheme. Note that, in Fig. 1(a), nodes 1 and 5 are equipped with single-hop grooming OXCs, which can only switch at wavelength granularity.

\section{B. Multihop Partial-Grooming OXC}

A multihop partial-grooming OXC consists of two switch fabrics, a wavelength-switch fabric (W-Fabric) which can be implemented using either transparent (all-optical) or opaque (electronic) technology, and an electronic-switch fabric which can switch low-speed traffic streams. The electronic-switch fabric is also called grooming fabric (G-Fabric). With this hierarchical switching and multiplexing architecture, this type of OXC can switch low-granularity traffic streams from one wavelength channel to other wavelength channels and groom them with other low-speed streams without using any extra network element. Assuming that the wavelength capacity is OC- $N$ and the lowest input port speed of the electronic switch fabric is OC- $M(N \geq M)$, the ratio between $N$ and $M$ is called the "grooming ratio." In this architecture, only a few of the wavelength channels (lightpaths) can be switched to the G-Fabric for switching at finer granularity. The number of ports, which connect the W-Fabric and G-Fabric, determines how much multihop grooming capability the OXC has. Fig. 2(a) shows a simplified multihop partial-grooming OXC architecture. A multihop partial-grooming OXC can support both single-hop grooming and multihop grooming schemes.

Fig. 1(a) also shows how a low-speed connection $(C 2)$ can be carried by multiple lightpaths $(L 1, L 2$, and $L 3)$ from node 1 to node 5 . Note that nodes 2 and 3 are equipped with multihop partial-grooming OXCs, and only their G-Fabrics are shown in the figure.

Fig. 1(a) also shows that there may exist four types of lightpaths in a WDM network which employs multihop partial-grooming OXCs. Assuming that, in Fig. 1(a), all network nodes are equipped with multihop partial-grooming OXCs, and only the G-Fabrics of nodes 2 and 3 and W-Fabrics of nodes 1,4 , and 5 are shown, lightpaths $L 1, L 2, L 3$, and $L 4$ represent these four lightpath types. 
- Multihop-ungroomable lightpath (L4): A lightpath $(i, j)$ is a multihop-ungroomable lightpath if it is not connected with a finer-granularity switching element at either of its end nodes. This lightpath can only be used to carry the traffic directly between node pair $(i, j)$. Lightpath $L 4$ in Fig. 1(a) is a multihop-ungroomable lightpath. For simplicity, this type of lightpath may also be called an ungroomable lightpath.

- Source-groomable lightpath (L3): A lightpath $(i, j)$ is a source-groomable lightpath if it is only connected with a finer-granularity switching element at its source node. All traffic on this lightpath has to terminate at node $j$, but the traffic may originate from any other network node as well. Lightpath $L 3$ in Fig. 1(a) is a source-groomable lightpath.

- Destination-groomable lightpath $(L 1)$ : A lightpath $(i, j)$ is a destination-groomable lightpath if it is only connected with a finer-granularity switching element at its destination node. All traffic on this lightpath has to originate from node $i$. At the lightpath destination node $j$, the traffic on lightpath $(i, j)$ can either terminate at $j$ or be groomed to other lightpaths and switched toward other nodes. Lightpath $L 1$ in Fig. 1(a) is a destination-groomable lightpath.

- Full-groomable lightpath (L2): A lightpath $(i, j)$ is a full-groomable lightpath if it connects to finer-granularity switching elements at both end nodes. This lightpath can be used to carry traffic between any node pair in the network. Lightpath $L 2$ in Fig. 1(a) is a full-groomable lightpath. Please note that, the source-groomable lightpath, destination-groomable lightpath, and full-groomable lightpath can be also called multihop-groomable lightpath.

In an optical WDM network employing multihop partial-grooming OXCs, the lightpaths can either be established dynamically according to current connection requests, or be preplanned based on forecasted traffic demands.

\section{Multihop Full-Grooming OXC}

A multihop full-grooming OXC can provide full-grooming functionality, i.e., every $\mathrm{OC}-\mathrm{N}$ wavelength channel arriving at the OXC will be demultiplexed into its constituent OC-M streams before it enters the switch fabric. The switch fabric can switch these OC-M traffic streams in a nonblocking manner. Then, the switched streams will be multiplexed back onto different wavelength channels. An OXC with full-grooming functionality has to be built using the opaque approach.

When a WDM network employs multihop full-grooming OXC at every network node, every wavelength channel on each fiber link forms a full-groomable lightpath between adjacent node pairs. Therefore, the virtual topology (i.e., lightpath topology) is predetermined and exactly the same as the physical topology (fiber topology). A low-speed traffic stream can be easily switched from one time slot of a wavelength channel to another time slot of a possibly different wavelength channel at every intermediate node which it traverses. A multihop full-grooming OXC can support both single-hop and multihop grooming schemes.

The time-space-time (TST) switching technology can be used to implement the multihop full-grooming OXC switch fabric.

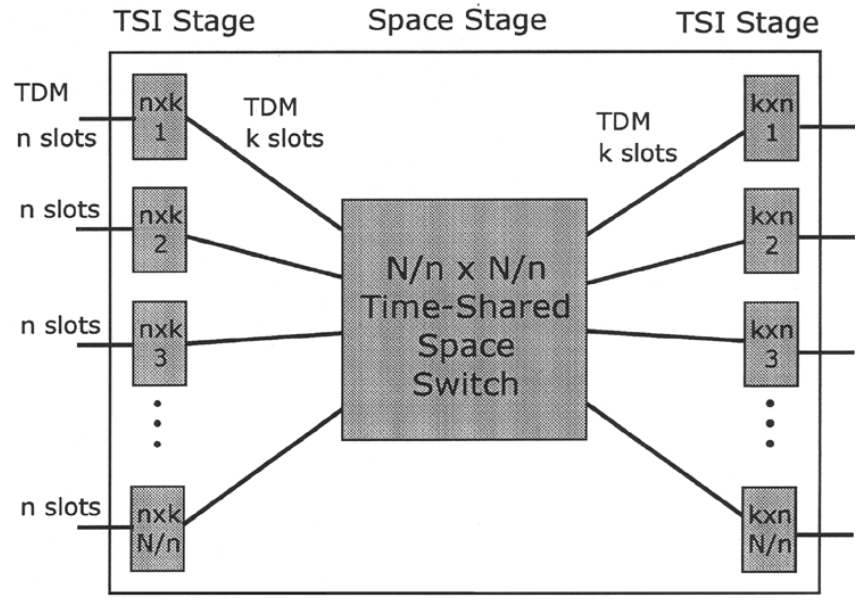

Fig. 3. Abstract overview of TST switch architecture.

Fig. 3 shows an overview of such a multistage switching architecture. Each input port of the fabric shown in Fig. 3 consists of $n$ time slots and the fabric can switch $N$ time slots in a nonblocking manner. Hence, the total number of input ports (as well as output ports) is $N / n$. Such a fabric consists of 3 stages. The first stage performs time-slot interchange (TSI) functionality, through which each of the $n$ time slots from an input port can be directed toward any of the $k$ time slots toward the second stage. The second stage performs space-switching functionality, through which $N / n$ traffic streams (one time slot from each input port) can be switched toward the third stage. Note that the second stage may only require a single space-switch component since, at any instance, there are at most $N / n$ traffic streams to be switched toward the third stage (one time slot from each input port). This architecture is called a time-shared-space switch. Again, the third stage performs time-slot interchange functionality. Based on the "Clos" switch architecture, in order to support nonblocking switching at lower granularity level (say, STS-1), the number of time slots supported by the internal connections (between stage 1 and 2, and between stage 2 and 3) should be nearly two times the number of time slots carried by the input/output ports (i.e., $k=2 n-1$ ). In other words, the internal speed of such a TST-based switch should nearly double the date rate of its input/output ports, which may lead to a significant technical difficulty to develop a large-scale multihop full-grooming OXC operating at high speed (e.g., OC-192, approximately $10 \mathrm{~Gb} / \mathrm{s}$ and OC-768, approximately $40 \mathrm{~Gb} / \mathrm{s}$ ).

\section{Light-Tree-Based Source-Node Grooming OXC}

Optical "light tree' has been proposed to support multicast applications in optical WDM networks [18], [19]. A light-tree is a wavelength tree which connects one source node to multiple destination nodes. Through a light tree, traffic from the source node will be delivered to all destination nodes of the tree. In a light tree, the node which originates the traffic is called the "root" node and the nodes which terminate the traffic are called the "leaf" nodes. Note that a leaf node can also serve as an intermediate multicast node since it can both receive traffic and forward the traffic to other nodes using its multicast capability. In order to support multicast, an OXC needs to duplicate the traffic from one input port to multiple output ports. For an OXC using 
TABLE I

Summary of the Characteristics OF DiFfERENT Optical GROOMING SWITCHES

\begin{tabular}{c|c|c|c|c|c|c|c|c|c|}
\hline Type \ Charac. & $\begin{array}{l}\text { Grooming } \\
\text { capability }\end{array}$ & $\begin{array}{l}\text { Provisioning } \\
\text { flexibility }\end{array}$ & $\begin{array}{c}\text { Switching } \\
\text { capacity }\end{array}$ & Cost & Scalability & $\begin{array}{c}\text { Optical } \\
\text { bypassing }\end{array}$ & $\begin{array}{c}\text { Technology maturity } \\
\text { (hardware) }\end{array}$ & $\begin{array}{c}\text { Technology maturity } \\
\text { (control software) }\end{array}$ \\
\hline $\begin{array}{c}\text { Single-hop } \\
\text { grooming } \\
\text { OXC }\end{array}$ & poor & poor & largest & low & good & can & medium & medium \\
$\begin{array}{c}\text { Source-node } \\
\text { grooming } \\
\text { OXC }\end{array}$ & good & good & largest & meduim & good & can & medium & low \\
$\begin{array}{c}\text { Multi-hop } \\
\text { partial-grooming } \\
\text { OXC }\end{array}$ & better & better & large & medium & good & can & medium & low \\
$\begin{array}{c}\text { Multi-hop } \\
\text { full-grooming } \\
\text { OXC }\end{array}$ & best & best & small & high & poor & cannot & high & high \\
\hline
\end{tabular}

transparent technology, this duplication can be realized in the optical domain using an optical splitter by splitting the power of an optical signal from one input port to multiple output ports. For an OXC using opaque technology, the traffic duplication can be easily accomplished by copying the electronic bit stream from one input port to multiple output ports. Fig. 2(b) shows a simplified architecture of a multicast-capable $\mathrm{OXC}$ using the transparent technology.

Fig. 1(b) shows how the OXCs' multicast capability can be used to perform traffic grooming. There are three low-speed traffic steams from the same source node 1 to different destination nodes 3,5 , and 6 . By setting up a light tree, these three traffic streams can be packed onto the same wavelength channel, and delivered to all destination nodes (i.e., light-tree leaf nodes). At each destination node, only the appropriate traffic stream is picked up and relayed to the client equipment. In this way, the low-speed traffic from the same source node can be groomed to the same wavelength channel and be sent to different destination nodes. We call this grooming scheme light-tree-based source-node grooming scheme. Please note that, if a connection between a node pair requires full wavelength-channel capacity, a light tree becomes a lightpath. From traffic-grooming perspective, the multicast-capable OXC can be called a lighttree-based source-node grooming OXC. Such an OXC can support the light-tree-based source-node grooming scheme as well as the single-hop grooming scheme.

\section{E. Summary of Different Grooming Schemes and Grooming OXC Architectures}

As a summary, the single-hop grooming scheme can only groom traffic from the same source node to the same destination node; the light-tree-based source-node grooming scheme can groom traffic from the same source node to different destination nodes; and the multihop grooming schemes may groom traffic from different source nodes and to different destination nodes.

Table I summarizes the characteristics of different optical grooming OXCs. The multihop full-grooming $\mathrm{OXC}$ has the best grooming capability and provisioning flexibility, but it can only be implemented using the opaque technology. Therefore, it requires a significant amount of electronic processing, which potentially leads to poor scalability and high cost (normalized per-bit switching cost). Since it has built-in wavelength-conversion capability and full-grooming capability, the network control of this OXC encounters less physical constraints and will be relatively easy to develop.

The single-hop grooming OXC, on the other hand, has poor grooming capability and does not have too much flexibility to provision connections of different bandwidth granularities, since only the single-hop grooming scheme is supported. Both transparent (less mature) and opaque (more mature) technologies can be used to develop this type of OXC. As the OXC always switches traffic at high granularity, it may have the largest switching capacity and the lowest cost (normalized per-bit switching cost). The single-hop grooming OXC with transparent technology can have good scalability (for wavelength-band switching or fiber switching). Depending on the implementation, the OXC may employ the wavelength-continuity constraint (if it is built with transparent technology and has no wavelength-conversion capability or only has partial wavelength-conversion capability). Hence, certain intelligent control algorithms are required. Provisioning connections in a WDM network with the wavelength-continuity constraint is known as a standard routing and wavelength assignment (RWA) problem and has been well addressed in the literature.

Most characteristics of the source-node grooming OXC and the multihop partial-grooming $\mathrm{OXC}$ are between those of the single-hop grooming $\mathrm{OXC}$ and the multihop full-grooming OXC. Intelligent algorithms are needed for WDM networks which employ light-tree-based source-node grooming OXCs (or multihop partial-grooming OXCs) to efficiently set up light trees (or multihop-groomable lightpaths). Compared with the RWA problem, there are relatively few references in the literature on this subject. More efforts are needed to investigate the traffic-grooming problem and to develop the novel algorithms, which can fully utilize the grooming capability of OXCs and optimize the network's resource efficiency.

\section{APPROACHES AND ALGORITHMS}

In this section, we present two approaches and algorithms to efficiently achieve the proposed grooming schemes in an optical WDM network, one for single-hop and multihop grooming schemes and the other for the light-tree-based source-node grooming scheme. 


\section{A. Single-Hop and Multihop Grooming Using an Auxiliary Graph Model}

1) Grooming Policies and an Auxiliary Graph Model: In a traffic-groomable WDM network, there may be multiple ways to carry a low-speed connection request, i.e., there may exist multiple routes from a given source node to a given destination node, each of which may use different amount of network resources, e.g., wavelength channels, grooming ports, etc. The decision on how to choose a proper route among multiple candidate routes is known as the network operator's "grooming policy." Different grooming policies reflect the network operator's intention on how to engineer their traffic using the available network resources. For example, a low-speed connection can be carried through existing lightpaths, by setting up a new lightpath between the given source-destination node pair, or by a combination of both existing lightpaths and newly established lightpaths. The effect of different grooming polices on network performance has been addressed in [12] and [16].

We extend a generic graph model, which was originally proposed in [12], to handle the single-hop grooming and the multihop grooming schemes. The extended model can uniformly incorporate different grooming OXC architectures (single-hop grooming $\mathrm{OXC}$, multihop partial-grooming $\mathrm{OXC}$, and multihop full-grooming $\mathrm{OXC}$ ) and can easily realize different grooming policies. In this model, an auxiliary graph is constructed for a given network state. The route of a connection request is computed based on the auxiliary graph. We use a graph $G(V, E)$ to represent a given network state, where $V$ denotes the network node set (i.e., the OXCs) and $E$ denotes the network link set, (i.e., the fiber links and the lightpath links). We use $G^{\prime}\left(V^{\prime}, E^{\prime}\right)$ to denote the corresponding auxiliary graph, where $V^{\prime}$ denotes the vertex set and $E^{\prime}$ denotes the edge set. From now on, for clarity, we will use the terms node and link to represent a vertex and an edge in the original network state graph $G(V, E)$, respectively, and the terms vertex and edge to represent a vertex and an edge in the auxiliary graph $G^{\prime}\left(V^{\prime}, E^{\prime}\right)$, respectively.

The auxiliary graph $G^{\prime}\left(V^{\prime}, E^{\prime}\right)$ can be divided into four layers, namely the access layer, the mux layer, the grooming layer, and the wavelength layer. The access layer represents the access points of a connection request, i.e., the points where a customer's connection starts and terminates. It can be an IP router, an ATM switch, or any other client equipment. The mux layer represents the OXC ports from which low-speed traffic streams are directly multiplexed (demultiplexed) onto (from) wavelength channels and switched by the W-Fabric without going through any G-Fabric. It can be an electronic multiplexer/demultiplexer, a SONET ADM, etc. The grooming layer represents the grooming component of the network node, e.g., the grooming fabric. The wavelength layer represents the wavelength-switching capability, i.e., the W-Fabric, and the link state of wavelength channels. A network node is divided into two vertices at each layer. These two vertices represent the input port and output port of the network node at that layer. Fig. 4 shows the graph representation of different grooming OXCs. For simplicity, we assume that every network node has full wavelength-conversion capability. The edges in Fig. 4 represent the resource availability at a given network

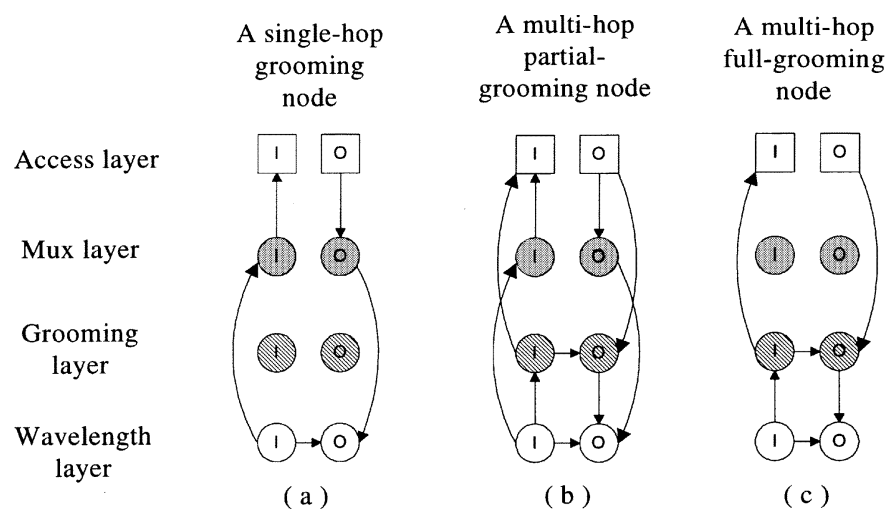

Fig. 4. Different grooming OXCs and their representations in the auxiliary graph.

node. They also denote the reachability from a given port of a given layer to another port of another layer in a network node. A connection request between node pair $(i, j)$ always originates from the output port of the access layer in node $i$ and terminates at the input port of the access layer in node $j$. Note that, through some straightforward extensions (by stretching the single-wavelength layer to multiple layers, one for each wavelength), the network without full wavelength-conversion capability can also be properly modeled.

The links in the original network state graph $G(V, E)$ can be represented by the edges which connect the vertices from one network node to another network node shown in Fig. 4. We explain them as follows.

- A wavelength link $(i, j)$ can be represented by an edge which connects the output port of the wavelength layer in node $i$ to the input port of the wavelength layer in node $j$.

- A multihop-ungroomable lightpath $(i, j)$ can be represented by an edge, which connects the output port of the mux layer in node $i$ to the input port of the mux layer in node $j$.

- A source-groomable lightpath $(i, j)$ can be represented by an edge, which connects the output port of the grooming layer in node $i$ to the input port of the mux layer in node $j$.

- A destination-groomable lightpath $(i, j)$ can be represented by an edge which connects the output port of the mux layer in node $i$ to the input port of the grooming layer in node $j$.

- A full-groomable lightpath $(i, j)$ can be represented by an edge which connects the output port of the grooming layer in node $i$ to the input port of the grooming layer in node $j$.

Fig. 5 illustrates how to construct the auxiliary graph for a given network state. Fig. 5(a) and (b) shows a network state for a simple 3-node network. The shaded node (node 0) employs a multihop partial-grooming OXC and the un-shaded nodes (nodes 1 and 2) are equipped with single-hop grooming OXCs. Each link in Fig. 5(a) represents a free wavelength channel between a node pair, and each link in Fig. 5(b) represents an established lightpath. The lightpath $(0,2)$ is a source-groomable lightpath, the lightpath $(1,0)$ is a destination-groomable lightpath, and the lightpath $(2,1)$ is a multihop-ungroomable lightpath. A low-speed connection request from node 1 to 


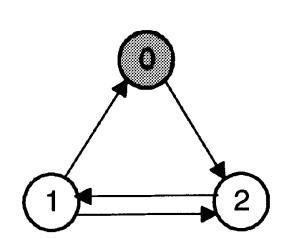

( a )

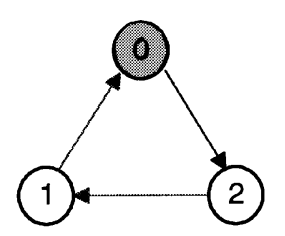

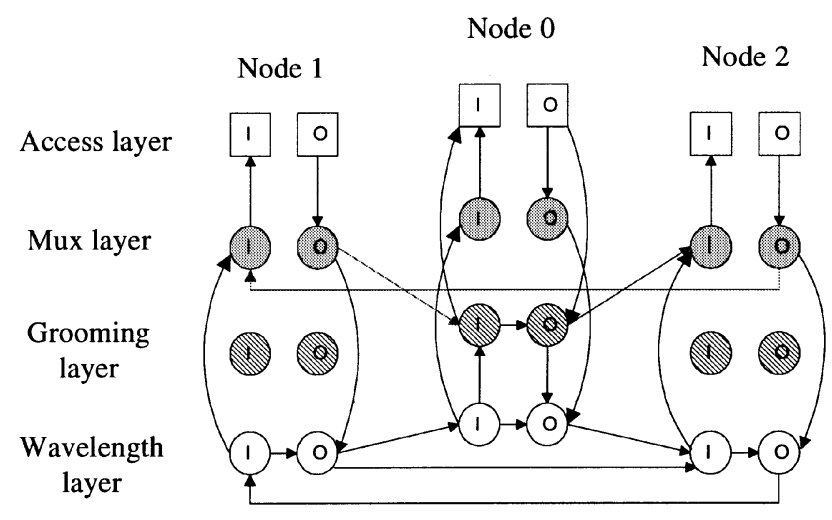

Fig. 5. Network state for a simple three-node network and the corresponding auxiliary graph.

node 2 can be carried by lightpaths $(1,0)$ and $(0,2)$. On the other hand, a request from node 2 to node 0 cannot traverse lightpaths $(2,1)$ and $(1,0)$ since node 1 does not have multihop grooming capability. According to the network state shown in Fig. 5(a) and (b), we can construct the auxiliary graph, which is shown in Fig. 5(c).

\section{2) Grooming Using Auxiliary Graph (GUAG) Algorithm:}

Grooming Using auxiliary Graph (GUAG) Input: Network state graph $G(V, E)$, and a connection request $\operatorname{Req}(s, d, c)$ where $s$ and $d$ denote the source and destination nodes of the request $(s, d \in V)$, and $c$ denotes the connection's capacity requirement.

Output: A route $R$ between nodes $s$ and $d$, which satisfies the connection's capacity requirement and a new network state $G_{n e w}\left(V_{\text {new }}, E_{\text {new }}\right)$ after provisioning the connection.

1) Construct the auxiliary graph $G^{\prime}\left(V^{\prime}, E^{\prime}\right)$ according to network resource availability and the bandwidth requirement of the request, i.e., (a) if there is no free wavelength on a fiber connecting a node pair $(i, j)$, there is no edge connecting the vertices of the wavelength layer between node pair $(i, j)$; (b) if there is no lightpath link between a node pair $(i, j)$, or lightpaths between $(i, j)$ do not have enough free capacity for Req, there is no corresponding edge in the auxiliary graph; and (c) if the multihop partial-grooming OXC at a given node $i$ has used up all grooming ports, there is no edge between the vertices of the grooming layer and the wavelength layer in node $i$.

2) Assign proper weights to each edge in $G^{\prime}$, according to the grooming policy. The grooming policy we use in this study is described as follows.

a) If there is any multihopungroomable lightpath between $(s, d)$ with enough free capacity, carry Req using this lightpath.

b) Otherwise, a connection is provisioned through the least-cost route. In this study, the cost of a fiber link is assumed to be unity. The cost of a lightpath link is equal to the overall cost of the concatenated fiber links it traverses.

c) If there are multiple least-cost routes and the connection does not require full wavelengthchannel capacity, choose the route which employs the minimal number of free wavelength links.

d) If there are multiple least-cost routes and the bandwidth requirement of the connection is full wavelength-channel capacity, choose the route which traverses the minimal number of G-Fabrics.

Please refer to [12] on how to assign proper edge weights to the auxiliary graph according to a grooming policy.

3) Compute a route $R^{\prime}$ based on the auxiliary graph $G^{\prime}$. If no path exists, return NULL.

4) Map $R^{\prime}$ in $G^{\prime}$ back to a route $R$ in the original network state graph $G$.

5) Allocate resource and update the network state according to the route $R$. It may include the operations of (a) updating the number of available wavelength on fiber links along the route $R$, if necessary; (b) creating new lightpath links if necessary; (c) updating the number of grooming ports in a partial- 


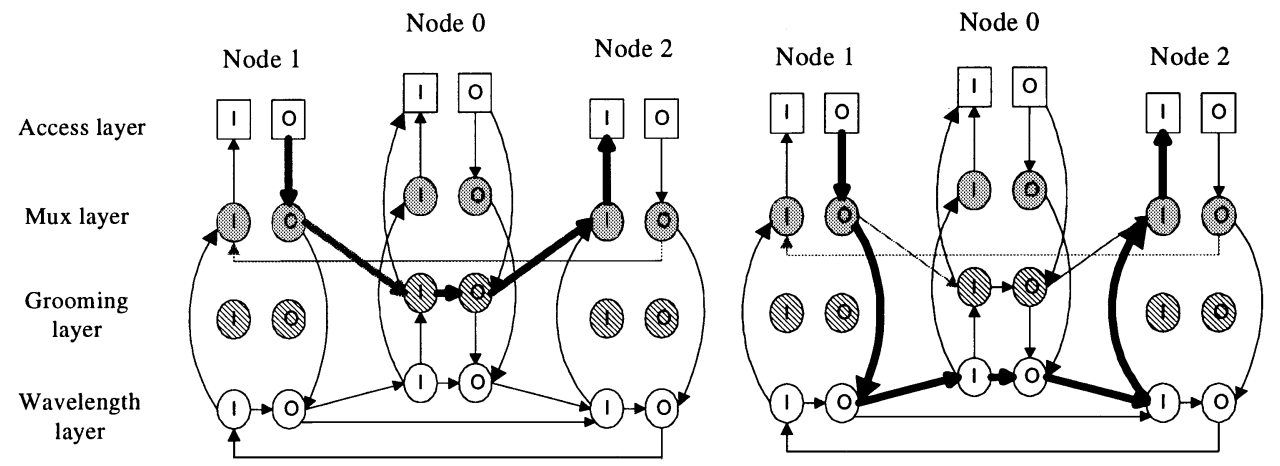

Fig. 6. Two alternative routes which can be found using the auxiliary graph.

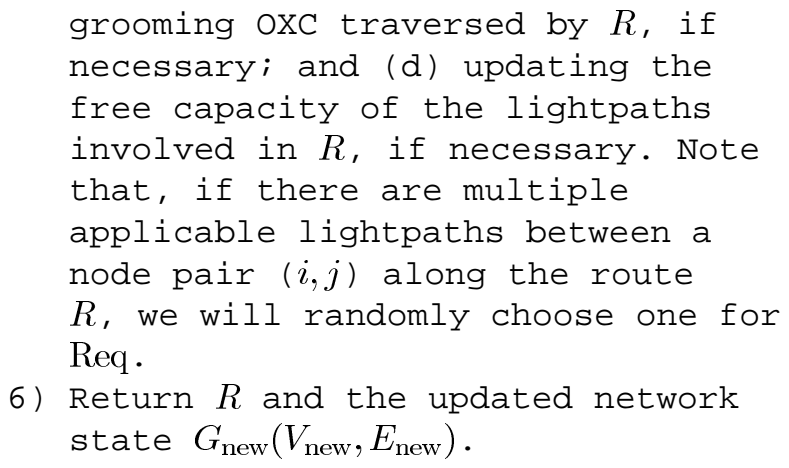

6) Return $R$ and the updated network state $G_{\text {new }}\left(V_{\text {new }}, E_{\text {new }}\right)$.

This auxiliary graph can be used to provision customers' connection requests. By assigning proper weights (i.e., administrative costs) to each edge in the auxiliary graph, suitable routes will be computed through standard shortest-path route-computation algorithms according to different grooming policies. Fig. 6 illustrates how to achieve different grooming policies by using the graph model. The network state and the auxiliary graph representation are shown in Fig. 5. Suppose that there is a new traffic request from node 1 to node 2. Fig. 6 shows two possible routes (in thick edges) for this connection request. The route shown in Fig. 6(a) traverses two existing lightpath links, while the route shown in Fig. 6(b) employs two new wavelength links. If the connection requires full wavelength-channel capacity, or if the overall bandwidth requirement of future demands between node pair $(1,2)$ is estimated to be close to full wavelength capacity, the route in Fig. 6(b) is preferred since the wavelength channels are fully utilized and no grooming is needed at node 0; otherwise, the route in Fig. 6(a) may be preferred if enough free capacity is available in the existing lightpaths. More detailed study on how to use this graph model to achieve different grooming policies and how these grooming policies may affect network performance can be found in [12]. Based on this model, we design an algorithm, called grooming using auxiliary graph (GUAG), which can be used to provision connections of different bandwidth granularities. This algorithm can be used in a WDM network which employs single-hop grooming OXCs, multihop partial-grooming OXCs, or multihop full-grooming OXCs.

3) Computational Complexity of GUAG: In GUAG, the time complexity to construct an auxiliary graph for a $N$-node, fullwavelength-convertible WDM network is $O\left(N^{2}\right)$, because the auxiliary graph of such a network consists of $2 \times 4 \times N$ vertices and at most $(2 \times 4 \times N)^{2}$ edges. Consequently, the computational complexity of Step 1 and Step 2 in algorithm (GUAG) is $O\left(N^{2}\right)$. Step 3 computes a least-cost route between two given vertices based on the auxiliary graph using standard shortest-path algorithms. Since the auxiliary graph has $2 \times 4 \times N$ nodes, the computational complexity of Step 3 is also $O\left(N^{2}\right)$. The computational complexity of the remaining steps in GUAG is $O(N)$. Therefore, the overall computational complexity of GUAG is $O\left(N^{2}\right)$ for a full-wavelength-convertible WDM network. Using the same analogy, we know that the computational complexity of GUAG will be $O\left(N^{2} W^{2}\right)$ in a wavelength-continuous WDM network, where $W$ is the number of wavelength channels supported by each fiber link.

\section{B. Source-Node Grooming Using Light-Tree Approach}

The proposed graph model and the GUAG algorithm cannot be used to handle the light-tree-based source-node grooming scheme. Therefore, we design another algorithm to perform efficient traffic grooming in a WDM network employing multicast-capable OXCs. Note that, in a WDM network using the light-tree-based source-grooming scheme, the network state can be represented as a graph $G(V, E, T)$, where $V$ denotes the network node set, $E$ denotes the network link set, and $T$ denotes the established light-tree set.

1) Grooming Using Light Tree (GULT) Algorithm: The proposed algorithm for the light-tree-based source-grooming scheme is described in algorithm 2 (GULT)

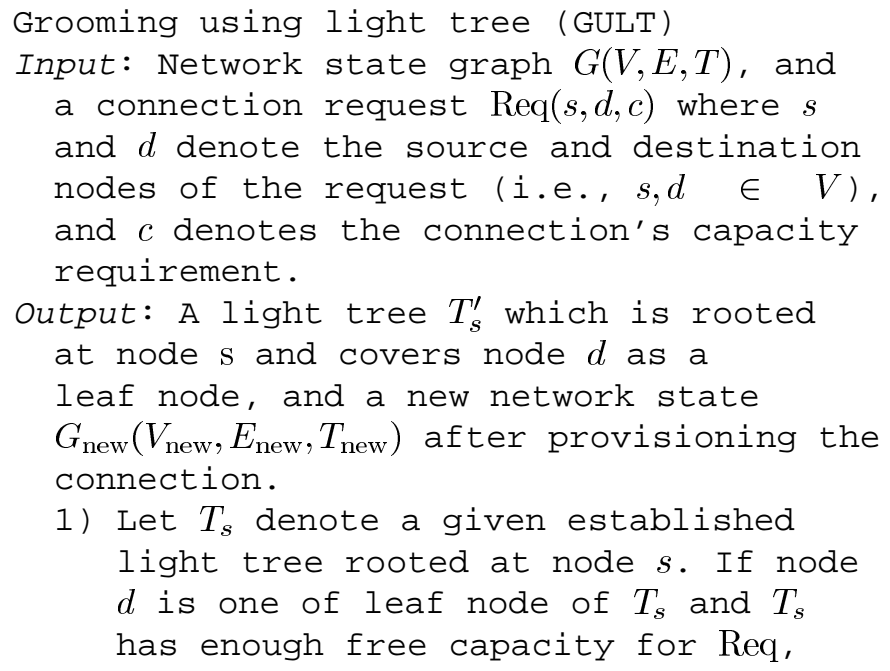


let $T_{s}^{\prime}$ be equal to $T_{s}$, and go to step 5. Note that, if multiple such trees exist, one will be randomly picked to be $T_{s}^{\prime}$.

2) For any $T_{s}$ which has enough free capacity for Req, compute a leastcost route from every tree node $j$ in $T_{s}$ to node $d$, subject to available wavelength-link constraints, and available splitter constraints at node $j$ (if the transparent technology is used to build the OXC at node $j$, as shown in Fig. 2(b)). Let $i$ denote the node which has the least-cost route to $d$ among all the tree nodes. If no such tree exists, go to step 4.

3) Find the $T_{s}^{\prime}$ and the corresponding node $i^{\prime}$ so that node $i^{\prime}$ is the closest one to node $d$ among all tree nodes of all candidate trees. If node $i^{\prime}$ is the root node of $T_{s}^{\prime}$, go to Step 4; otherwise, expand the tree to include a new branch from node $i^{\prime}$ to node $d$. Therefore, node $i^{\prime}$ needs to duplicate the traffic originating from node $s$ and switch it to node $d$. After that, go to step 5.

4) Set up a light tree from node $s$ to node $d$ following the leastcost route based on the current network state. Note that this special light tree instance only has one leaf (destination) node. Hence, it is equivalent to a lightpath. Let this light tree be $T_{s}^{\prime}$.

5) Allocate free capacity of $T_{s}^{\prime}$ to Req. If $T_{s}^{\prime}$ is a new tree or if $T_{s}^{\prime}$ is a established tree but a new branch has been added, update the corresponding wavelength-link availability information.

6) Return $T_{s}^{\prime}$ and the new network state $G_{\text {new }}\left(V_{\text {new }}, E_{\text {new }}, T_{\text {new }}\right)$.

2) Computation Complexity of GULT: In Step 2 of GULT, the computational complexity to find the closest node $i$ to the destination node $d$ in a given light tree $T_{s}$ is $O\left(N^{2}\right)$. This is because node $i$ can be found by constructing a shortest-path tree $T_{d}$ rooted at node $d$. In $T_{d}$, we can easily find the closest node (to node $d$ ) which is also in tree $T_{s}$. Assuming that there are $K$ candidate light trees which are rooted at node $s$ and can be expanded to support the connection request, the computational complexities for Steps 2 and 3 will be $O\left(K N^{2}\right)$. The computational complexity of the other steps in GULT is $O(N)$. Hence, the overall computational complexity of GULT is $O\left(K N^{2}\right)$ in a full wavelength-convertible network. In a wavelength-continuous network, GULTs computational complexity

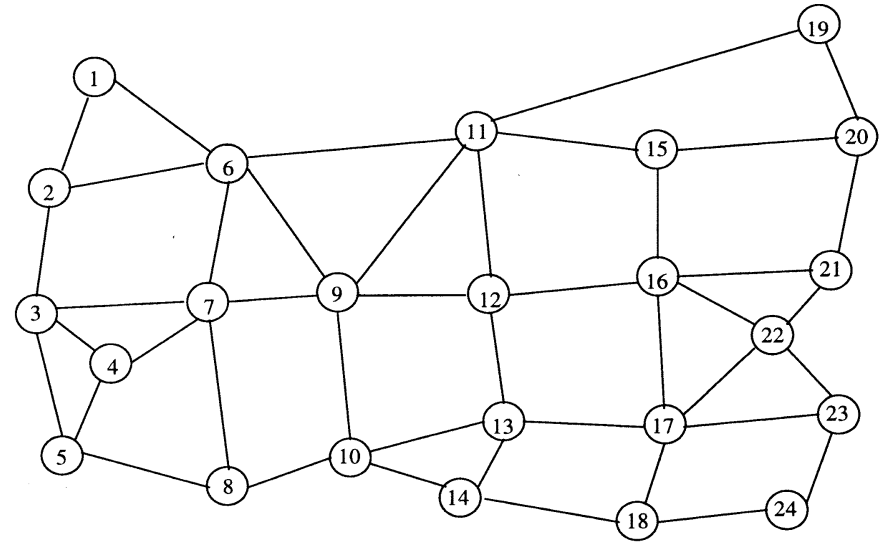

Fig. 7. 24-node sample network topology.

is $O\left(K W N^{2}\right)$, where $W$ is the number of wavelengths supported by a fiber link.

\section{ILLUSTRATIVE NUMERICAL Results}

We simulate a dynamic network environment to evaluate the performance of different optical grooming OXCs and their corresponding grooming schemes, using the proposed algorithms GUAG and GULT. The following parameters are used in our example study reported here.

- Every network node is equipped with the same type of grooming OXCs.

- The connection-arrival process is Poisson and the connection-holding time follows a negative exponential distribution.

- The capacity of each wavelength is OC-192.

- The network has full wavelength-conversion capability and each fiber link can support eight wavelength channels.

- A multihop partial-grooming OXC has six (incoming and outgoing) grooming ports.

- A light-tree-based source-node grooming OXC is built using the opaque technology and, hence, has unlimited multicast capability.

- A connection request can have any bandwidth granularity of $O C-1, O C-3, O C-12, O C-48$, and $O C-192$. Three bandwidth-granularity distributions for the number of connection requests $(O C-1: O C-3: O C-12: O C-$ 48:OC - 192) are examined, a) $3: 3: 3: 3: 1$, b) $1: 1: 1: 1: 1$, and c) $1: 1: 1: 1: 3$.

- Connections are uniformly distributed among all node pairs.

- Average connection-holding time is normalized to unity; network offered load (in Erlang) is defined as the connection-arrival rate times the average holding time times a connection's average bandwidth and it is normalized to the unit of OC-192.

- The example network topology used in our simulation experiments is shown in Fig. 7.

\section{A. Bandwidth Blocking Ratio (BBR)}

Bandwidth blocking ratio (BBR) represents the percentage of the amount of blocked traffic over the amount of bandwidth 

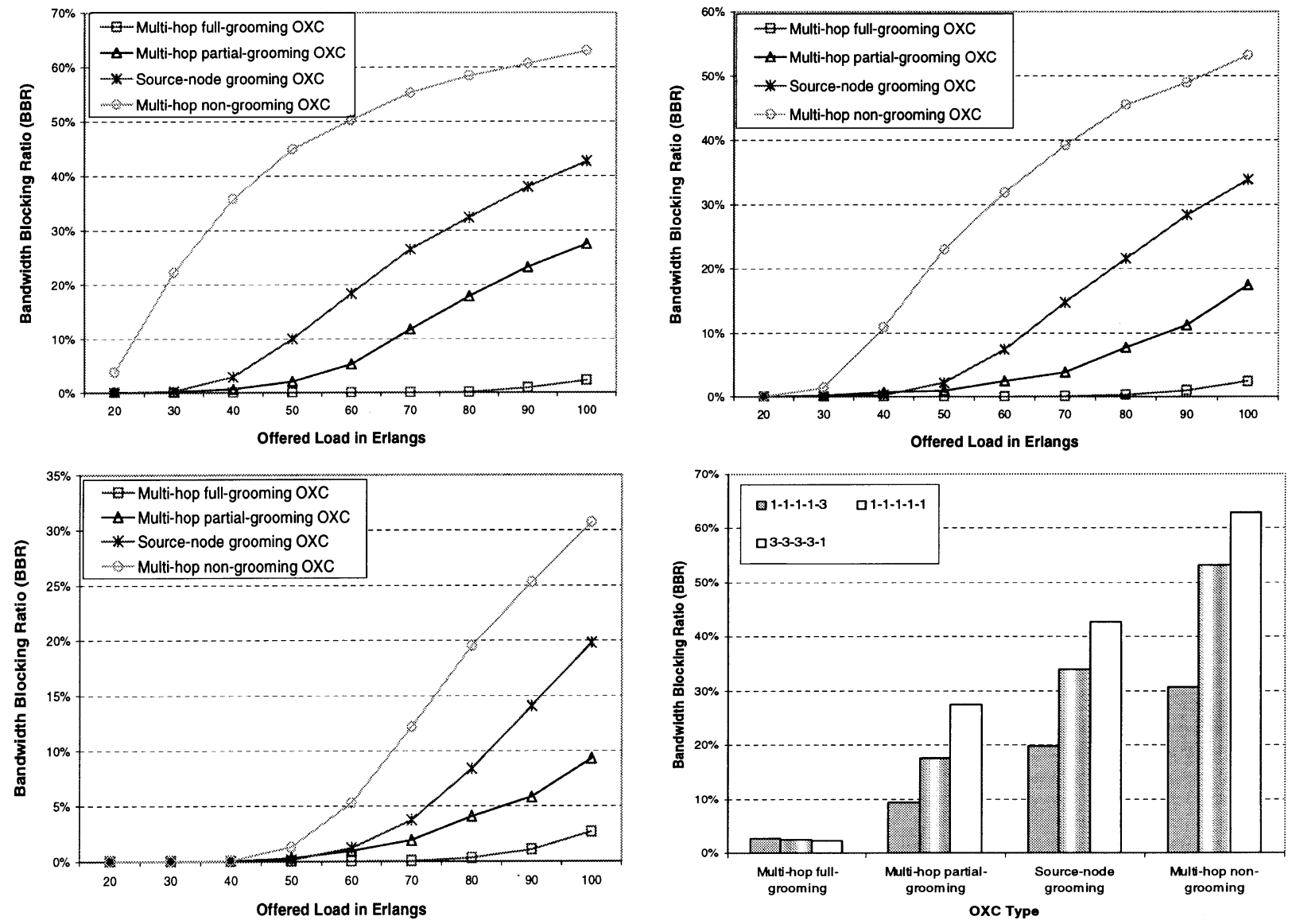

Fig. 8. BBR versus load (in Erlangs) for different grooming OXCs under different bandwidth-granularity distributions.

requirement of all traffic requests during the entire simulation period. Note that pure blocking probability cannot reflect the effectiveness of the algorithm, as connections have different bandwidth requirements.

Fig. 8 compares the network performance (BBR versus load) by using different optical grooming OXCs under different connection bandwidth-granularity distributions. In Fig. 8, the multihop full-grooming OXC shows the best network performance, and multihop nongrooming OXC shows the worst performance. We make the following observations.

1) Without any low-granularity multiplexing and switching functionality, the light-tree-based source-node grooming OXCs can significantly improve the network performance compared with multihop nongrooming OXCs.

2) Connection bandwidth-granularity distribution plays an important role on the network performance. When there are a lot of low-speed connection requests, multihop fullgrooming OXCs outperform multihop partial-grooming OXCs as shown in Fig. 8(a). As the number of high-speed connections increases, the performance gap between the multihop full-grooming $\mathrm{OXC}$ and the partial-grooming OXC is significantly reduced. Although not shown here, our experimented results verify that, when all connections require full wavelength-channel capacity, all OXCs have the same network performance. Note that the high BBR region shown in Fig. 8 may not be realistic in a practical WDM backbone network. They are shown for illustration purposes to fairly compare the grooming OXCs' network performance under the same network load.

3) As shown in Fig. 8(d), the multihop full-grooming OXC performs almost the same under different bandwidth-granularity distributions. On the other hand, under the same network load, if there are more low-speed connections, other types of grooming OXCs lead to worse network performance. This is because low-speed connections may potentially cause a large bandwidth waste and underutilize the wavelength capacity if a network node does not have multihop full-grooming capability.

As we can see from Fig. 8, compared with the multihop full-grooming OXC, the multihop partial-grooming OXC shows reasonably good network performance, while using significantly less amount of low-speed electronic processing. Besides the grooming policy and the corresponding grooming algorithm used, the performance of multihop partial-grooming OXCs is determined by two factors.

- How much grooming capacity a multihop partial-grooming OXC may have?

- How to cost-effectively establish the multihop-groomable lightpath (i.e., source-groomable lightpath, destination groomable-lightpath, and full-groomable lightpath) to perform multihop grooming?

One approach to improve the performance of the multihop partial-grooming OXC is to increase its grooming capacity. Recall that the grooming capacity of a multihop partial-grooming 


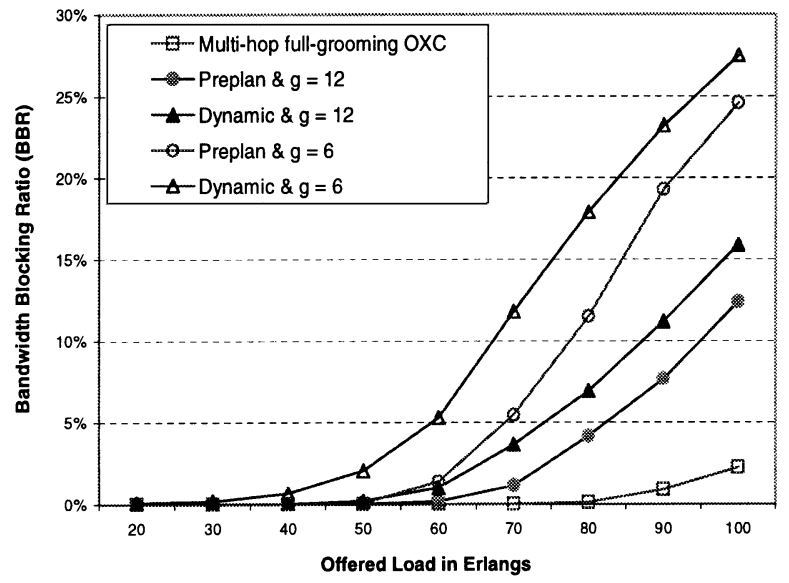

(a)

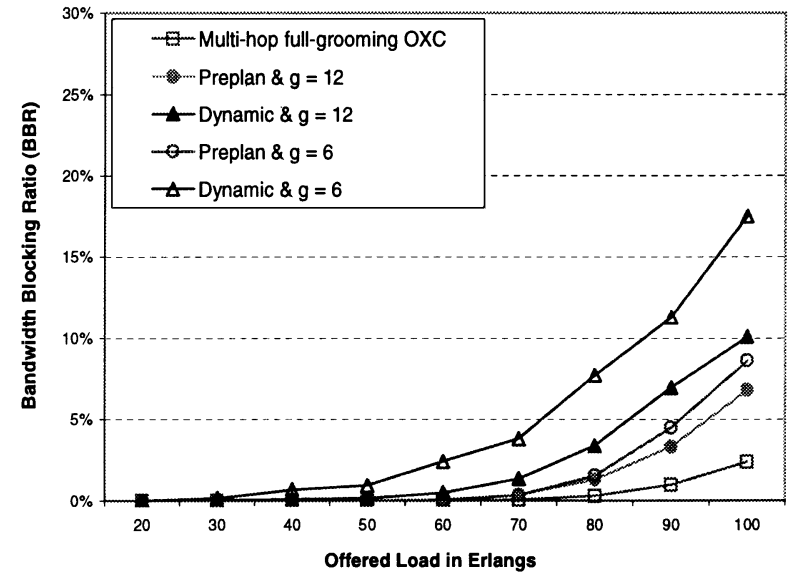

(b)

Fig. 9. Effect of different lightpath-establishment schemes and different number of grooming ports on the network performance of multihop partial-grooming OXCs.

OXC is determined by the size of its G-Fabric and the number of grooming ports (G-ports) connecting the W-Fabric and the G-Fabric. Another approach is to optimize the establishment of multihop-groomable lightpaths. These lightpaths can either be dynamically set up (on-demand) or be statically preplanned. In the dynamic groomable-lightpath establishment approach, it is assumed that future traffic demands are unknown. Hence, instead of considering long-term global optimization, groomable lightpaths are set up according to the requirement of the current connection request. On the other hand, the preplanning approach tries to preestablish a certain number of groomable lightpaths based on the future traffic projection. Low-speed connection requests are then dynamically provisioned using these preplanned resources. When all preplanned groomable lightpaths have been saturated, new groomable lightpaths can be dynamically established for new requests subject to network resource constraints.

Fig. 9 shows how the number of grooming ports and different groomable-lightpath establishment approaches may affect the network performance of multihop partial-grooming OXCs under different connection bandwidth-granularity distributions. In Fig. 9, $g$ denotes the number of grooming ports which a multihop partial-grooming OXC has. We observe that, as $g$ increases, the performance of the multihop partial-grooming OXC improves. We have also simulated a very simple lightpath preplanning scheme, called embedded on physical topology (EPT). In EPT, full-groomable lightpaths are preestablished between every adjacent node pair. These lightpaths form a grooming layer, which has exactly the same topology as the physical fiber topology. A low-speed connection will be carried by jointly utilizing the resources on this grooming layer as well as on physical topology through the grooming policies described in GUAG. Unlike a dynamically established lightpath which will be torn down if it does not carry any traffic, a preplanned-groomable lightpath will never be torn down. If one wavelength channel on each fiber link is used for EPT, we called it an one degree EPT (1-EPT), which is simulated in our experiments. Fig. 9 shows that the 1-EPT preplanning scheme does improve the performance of multihop partial-grooming OXC compared with the dynamic groomable-lightpath establishment scheme. This is because the dynamic scheme may greedily establish groomable lightpaths without considering the possible future traffic demands. Although the GUAG algorithm attempts to perform local (or short-term) resource optimization, the grooming layer, which is formed by dynamically-established lightpaths, may not be optimal and efficient to carry the future requests. If every multihop partial-grooming $\mathrm{OXC}$ has enough grooming capability (i.e., enough grooming ports) and $W$-EPT is used (where $W$ is the number of wavelength channels supported by every fiber link), a network employing multihop partial-grooming OXCs will be equivalent to a network with multihop full-grooming OXCs everywhere. Hence, they will have the same network performance.

Note that, besides the grooming policy used in GUAG, applying other grooming policies may further improve the network performance. Refer to [12] and [13] for other possible grooming policies and their effect on network performance. Similarly, besides 1-EPT, other preplanning schemes can also be used and it is possible for them to improve the network performance. For example, the integer linear program (ILP) formulation proposed in [10] can be used to preplan groomable lightpaths, and may achieve better network performance. A potential drawback of the preplanning approach is that it may not be scalable. This is because network operators may have to redo the preplan procedure when the network needs to be scaled or when the traffic pattern fluctuates. The effect of other preplanning schemes (2-EPT, ILP approach, etc.) is beyond the scope of this paper, and will be addressed in our future study.

\section{B. Wavelength Utilization (WU)}

Wavelength utilization (WU) represents the average number of used wavelength links over the total number of wavelength links supported by the network during the entire simulation period.

In Fig. 10, we plot the average (weighted by time) WU versus the network-offered load for different grooming OXCs under different connection bandwidth-granularity distributions. It is straight forward to see that, under the same network offered load, single-hop grooming OXCs will exhaust wavelength links 


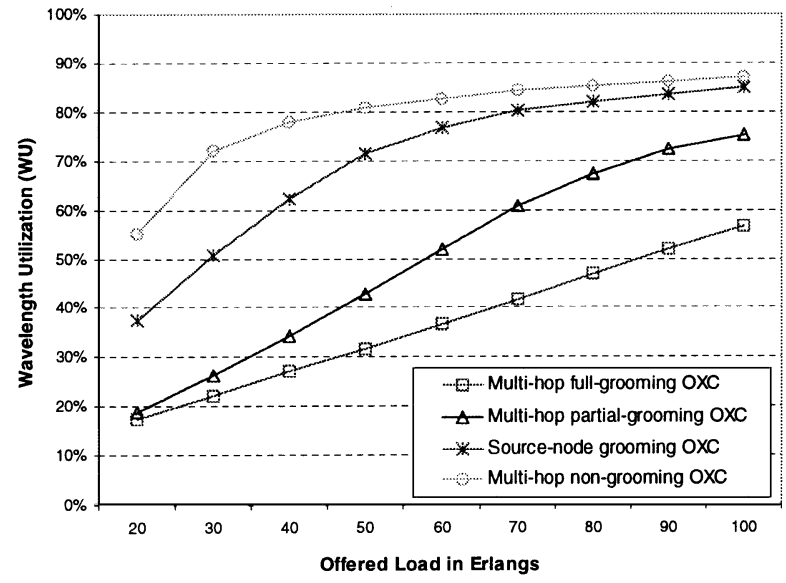

(a)

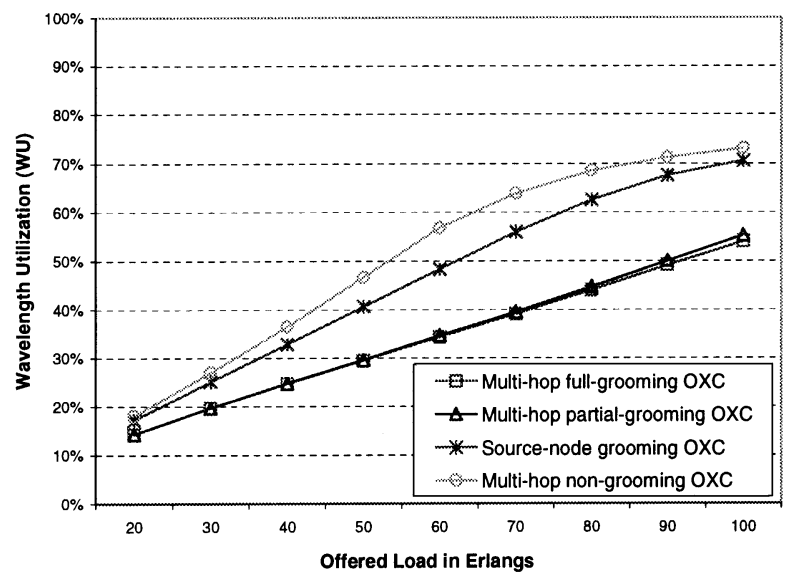

(c)

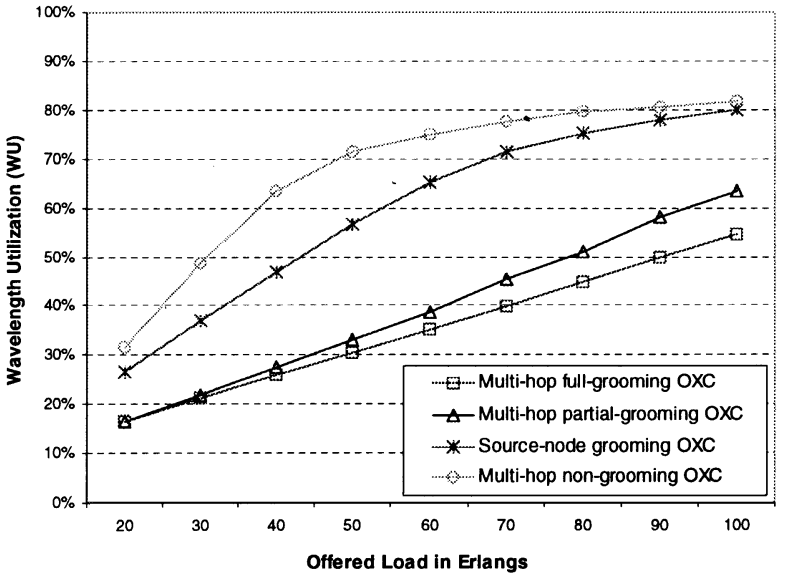

(b)

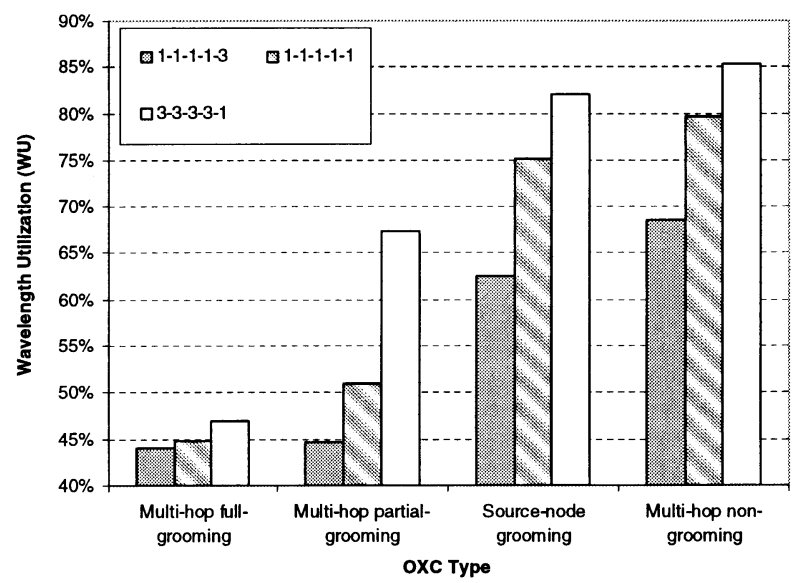

(d)

Fig. 10. Wavelength utilization (WU) versus load (in Erlangs) for different grooming OXCs under different bandwidth-granularity distributions.

more quickly than other OXC types. We can also observe from Fig. 10(d) that, under the same network offered load, the more low-speed connections the network supports, the more wavelength links tend to be used. For the same bandwidth-blocking performance, a lower wavelength utilization is desirable since fewer wavelength links are consumed to carry the same load.

\section{Resource Efficiency Ratio (RER)}

Wavelength utilization shown in Fig. 10 may not be the best metric to measure the resource usage of different grooming schemes and grooming OXCs. From Fig. 10, one cannot tell that how efficiently the allocated wavelength channels are utilized. RER is a more suitable metric to measure the grooming performance of different OXCs and different grooming schemes. RER represents how efficiently connections are routed and groomed. It can be computed as the average (weighted by time) network carried traffic (in terms of OC-1 units) divided by the total allocated network capacity (i.e., total number of allocated wavelength links times 192) over the entire simulation period. If we consider "minimal hops" as our objective for a route-computation algorithm, then the inverse of the average hop distance is the RER upper bound. This upper bound is achieved only when every connection requires OC-192 bandwidth and follows the shortest path. Since there are limited resources (as in our case), not every connection can follow the shortest path and the upper bound may not be achievable. Let $\alpha$ denote the RER. $\alpha$ can be computed as follows:

$$
\alpha=\frac{\sum_{i} \rho_{i} \times t_{i}}{\sum_{i} \gamma_{i} \times t_{i}}
$$

where $t_{i}$ is the time period between the $i^{\text {th }}$ event (connection arrival or departure) and $(i+1)^{t h}$ event, $\rho_{i}$ is the network carried load during the time period $t_{i}$, and $\gamma_{i}$ is the total number of wavelength links used during $t_{i}$. (Please note that $\rho_{i}$ and $\gamma_{i}$ do not change during time period $t_{i}$ as there is no other event during that period.)

Fig. 11 shows the normalized (to upper bound) RER versus network offered load for different grooming OXCs under different bandwidth-granularity distributions. The higher RER means that a network can route and groom traffic requests more efficiently. Thus, a network with high RER can have low BBR, which is shown in Fig. 8. This explains why, under the same network offered load, the multihop full-grooming OXC uses the least number of wavelength channels (i.e., the lowest wavelength utilization) but carries the most amount of traffic (i.e., the lowest bandwidth blocking ratio). We can also observe from Fig. 11 that, under the same network offered load, when the number of low-speed connections increases, the resource efficiency ratio will decrease. This is because the network 


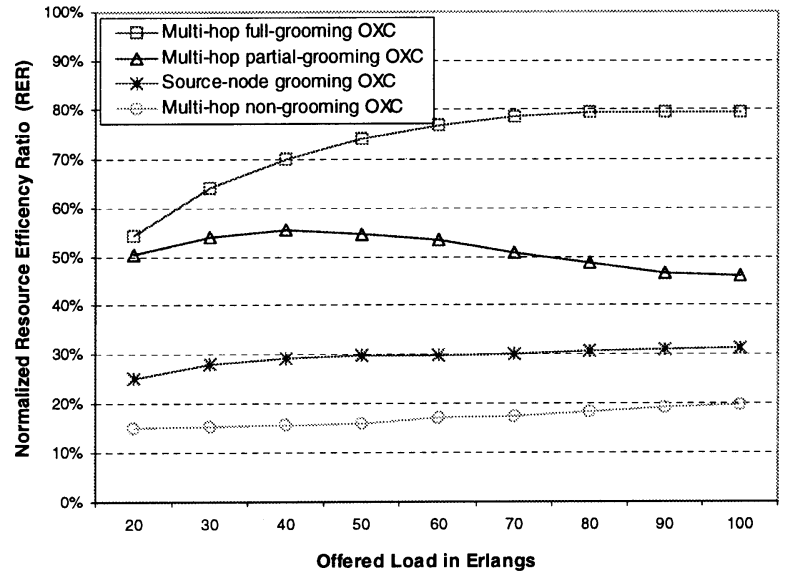

(a)

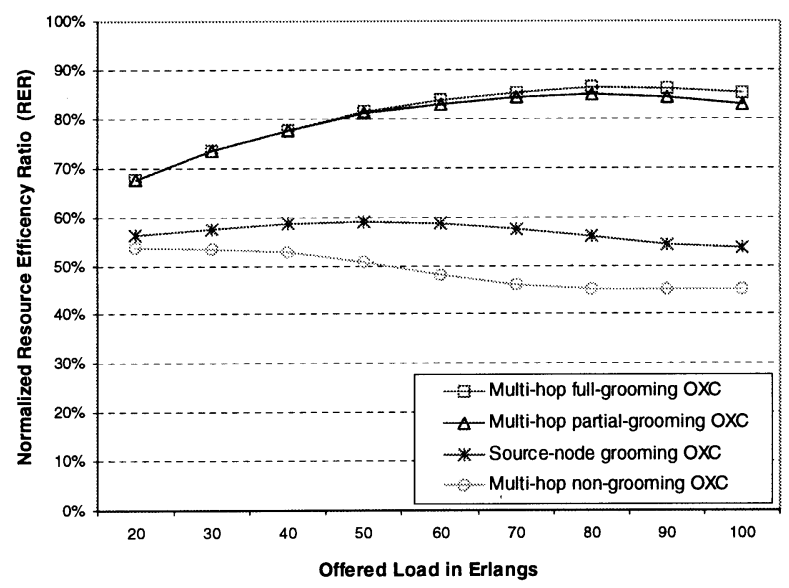

(c)

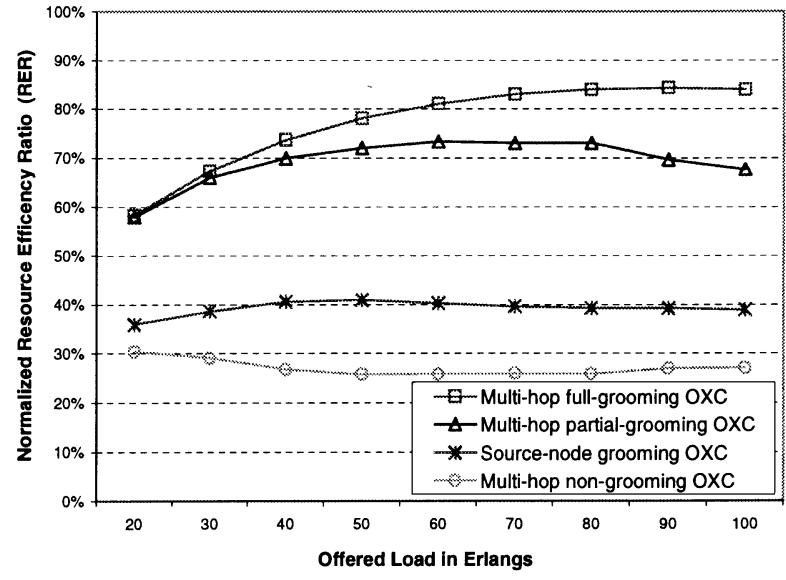

(b)

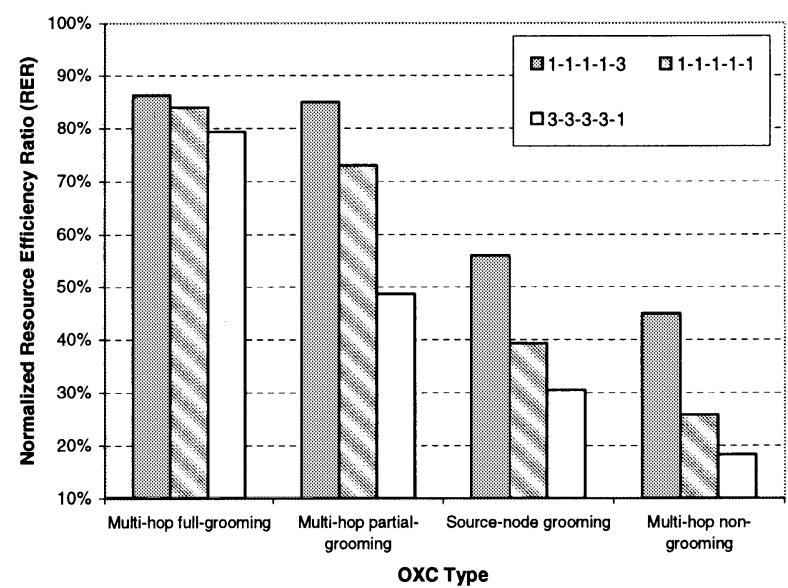

(d)

Fig. 11. Normalized RER versus load (in Erlangs) for different grooming OXCs under different bandwidth granularity distributions.

resources tend to be fragmented while the number of low-speed connections increases, and the difficulty for a network to fully utilize allocated wavelength channels also increases.

\section{CONCLUSION}

In this study, we presented four optical grooming OXC architectures and compared their characteristics. Three grooming schemes, single-hop grooming, multihop grooming, and light-tree-based source-node grooming for these OXCs were explored. We proposed two algorithms, GUAG and GULT, to efficiently provision connections of different bandwidth granularities. The performance of different grooming OXCs and grooming schemes was compared using the proposed algorithms under a dynamic traffic environment. We also investigated the effect of different bandwidth-granularity distributions to the network performance of different grooming OXCs. We observed that the light-tree-based source-node grooming using the OXCs' multicast capability can significantly improve the network performance of the single-hop grooming OXC without employing any low-granularity electronic processing. The multihop full-grooming OXC showed the best network performance in terms of network bandwidth blocking ratio, wavelength utilization, and resource efficiency ratio. However, it may encounter the scalability problem since a large amount of electronic processing is required at low-speed granularity. With a few nodes having low-granularity switching capability, the multihop partial-grooming OXC showed good performance compared with other grooming OXCs, which makes it as a good alternative when the multihop full-grooming is not needed at every network node. In order to fairly compare their performance, we assumed that a network uniformly employs one type of grooming OXCs. This assumption may not always be practical and can be relaxed in next-generation optical backbone networks, where different OXCs with different grooming capabilities may be interconnected and may need to co-exist. The proposed algorithms, GUAG and GULT, employed one traffic-grooming policy and light-tree establishment approach. Other algorithms with different traffic-grooming policies and light-tree establishment approaches will be addressed in our future research work.

\section{REFERENCES}

[1] B. Mukherjee, Optical Communication Networks. New York: McGraw-Hill, 1997.

[2] I. Chlamtac, A. Ganz, and G. Karmi, "Lightpath communications: An approach to high bandwidth optical WAN's," IEEE Trans. Commun., vol. 40, pp. 1171-1182, July 1992.

[3] O. Gerstel, P. Lin, and G. Sasaki, "Combined WDM and SONET network design," in Proc. INFOCOM '98, vol. 2, San Francisco, CA, Mar. 1998, pp. 734-743. 
[4] O. Gerstel, R. Ramaswami, and G. H. Sasaki, "Cost-effective traffic grooming in WDM rings," IEEE/ACM Trans. Networking, vol. 8, pp. 618-630, Oct. 2000.

[5] X. Zhang and C. Qiao, "On scheduling all-to-all personalized connections and cost-effective designs in WDM rings," IEEE/ACM Trans. Networking, vol. 7, pp. 435-443, June 1999.

[6] A. L. Chiu and E. H. Modiano, "Traffic grooming algorithms for reducing electronic multiplexing costs in WDM ring networks," J. Lightwave Technol., vol. 18, pp. 2-12, Jan. 2000.

[7] J. Wang, W. Cho, V. R. Vemuri, and B. Mukherjee, "Improved approaches for cost-effective traffic grooming in WDM ring networks: ILP formulations and single-hop and multihop connections," J. Lightwave Technol., vol. 19, pp. 1645-53, Nov. 2001.

[8] P. J. Wan, G. Calinescu, and O. Frieder, "Grooming of arbitrary traffic in SONET/WDM BLSR's," IEEE J. Select. Areas Commun., vol. 18, pp. 1995-2003, Oct. 2000.

[9] J. Simmons, E. Goldstein, and A. Saleh, "Quantifying the benefit of wavelength add-drop in WDM rings with independent and dependent traffic," J. Lightwave Technol., vol. 17, pp. 48-57, Jan. 1999.

[10] K. Zhu and B. Mukherjee, "Traffic grooming in an optical WDM mesh network," IEEE J. Select. Areas Commun., vol. 20, pp. 122-133, Jan. 2002.

[11] R. Ranganathan, J. Berthold, L. Blair, and J. Berthold, "Architectural implications of core grooming in a 46-node USA optical network," in Proc. IEEE/OSA OFC '02, Mar. 2002, pp. 498-499.

[12] H. Zhu, H. Zang, K. Zhu, and B. Mukherjee, "A novel, generic graph model for traffic grooming in heterogeneous WDM mesh networks," IEEE/ACM Trans. Networking, vol. 11, pp. 285-299, Apr. 2003.

[13] _ "A novel graph model for dynamic traffic grooming in WDM mesh networks," presented at the Proc. GLOBECOM '02, Taipei.

[14] S. Thiagarajan and A. K. Somani, "Capacity fairness of WDM networks with grooming capability," Opt. Networks Mag., vol. 2, no. 3, pp. 24-31, May/June 2001

[15] C. Xin, Y. Ye, S. Dixit, and C. Qiao, "An integrated lightpath provisioning approach in mesh optical networks," in Proc. IEEE/OSA OFC '02, Mar. 2002, pp. 547-549.

[16] K. Zhu and B. Mukherjee, "On-line provisioning connections of different bandwidth granularities in WDM mesh networks," in Proc. IEEE/OSA OFC '02, Mar. 2002, pp. 549-551.

[17] R. Srinivasan and A. K. Somani, "A generalized framework for analyzing time-space switched optical network," IEEE J. Select. Areas Commun., vol. 20, pp. 202-215, Jan. 2002.

[18] L. H. Sahasrabuddhe and B. Mukherjee, "Light-trees: Optical multicasting for improved performance in wavelength-routed networks," IEEE Commun. Mag., vol. 37, pp. 67-73, Feb. 1999.

[19] N. K. Singhal and B. Mukherjee, "Architectures and algorithms for multicasting in WDM optical mesh networks using opaque and transparent optical crossconnects," in Proc. IEEE OFC' 00 , Mar. 2001, p. TuG8.

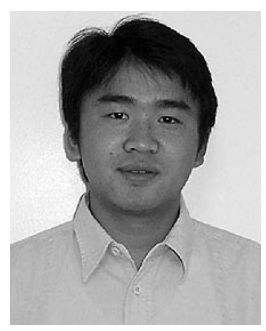

Keyao Zhu (S'98) received the B.S. degree in computer science from Peking University, Beijing, China, in 1998, and the M.S. and Ph.D. degrees in computer science from the University of California, Davis, in July 2000 and September 2003, respectively.

$\mathrm{He}$ is currently working at Research and Innovation Center, Alcatel Shanghai Bell Company, Ltd., China. His research interests include wavelength-routed WDM network design and analysis, WDM network protection, restoration, and traffic grooming, network security, and access networks.

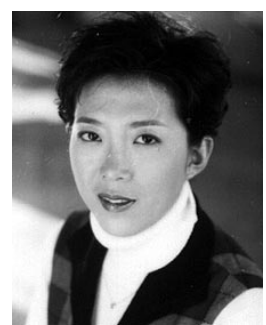

Hui Zang (S'97-M'02) received the B.S. degree in computer science from Tsinghua University, Beijing, China, in 1997, and the M.S. and Ph.D. degrees in computer science from the University of California, Davis, in 1998 and 2001, respectively.

In 2000, she joined Sprint Advanced Technology Laboratories, Burlingame, CA, where she is a principal applied Research and Development Engineer. She is the author of WDM Mesh Networks-Management and Survivability (Norwell, MA: Kluwer, 2002). She has published over 30 conference papers and journal articles and currently has three U.S. patents pending in the field of optical networking. Her research interests include WDM optical networks, IP over WDM networks, multiservice platforms, and quality-of-service.

Dr. Zang serves or has served as a Technical Committee Member of IPoW 2000-2002, APOC 2002, DRCN 2003, OptiComm 2003, and GLOBECOM 2003 Optical Networking and Systems Symposium. She helped organize OptiComm 2002 as a Panel Co-Chair. She was one of the Guest Editors of the IEEE Network special issue on Traffic Engineering in Optical Networks.

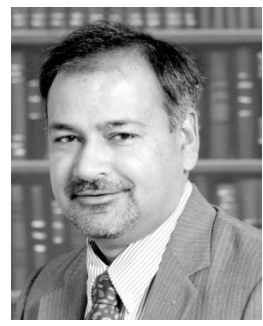

Biswanath Mukherjee (S'82-M'83) received the B.Tech. (honors) degree from Indian Institute of Technology, Kharagpur, in 1980 and the Ph.D. degree from University of Washington, Seattle, in June 1987.

He held a GTE Teaching Fellowship and a General Electric Foundation Fellowship at University of Washington. In July 1987, he joined the University of California, Davis, where he has been a Professor of computer science since July 1995, and served as Chairman of computer science from September 1997 to June 2000. He is the author of Optical Communication Networks (New York: McGraw-Hill, 1997) a book which received the Association of American Publishers, Inc.'s 1997 Honorable Mention in Computer Science. He is a Member of the Board of Directors of IPLocks, Inc., a Silicon Valley startup company. His research interests include lightwave networks, network security, and wireless networks.

Dr. Mukherjee is co-winner of Paper Awards presented at the 1991 and the 1994 National Computer Security Conferences. He serves or has served on the Editorial Boards of the IEEE/ACM TRANSACTIONS ON NETWORKING, the IEEE Network, ACM/Baltzer Wireless Information Networks (WINET), Journal of High-Speed Networks, Photonic Network Communications, and Optical Network Magazine. He also served as Editor-at-Large for optical networking and communications for the IEEE Communications Society. He served as the Technical Program Chair of the IEEE INFOCOM'96 Conference. He has consulted for and served on the Technical Advisory Board of a number of startup companies in optical networking. 